Universidad de los Andes

Facultad de Ciencias

Departamento de Matemática

On representation theorems for nonmonotonic consequence relations

Ramón Pino Pérez and Carlos Uzcátegui

Notas de Matemática

Serie: Pre-Print

No. 174

Mérida - Venezuela

1998 . 
Notas de Matemática, No. 174

Mérida, May 1998.

\title{
On representation theorems for nonmonotonic consequence relations
}

\author{
Ramón Pino Pérez and Carlos Uzcátegui
}

\begin{abstract}
Abstracit
One of the main tools in the study of nonmonotonic consequence relations is the representation of such relations in terms of preferential models. In this paper we give an unified and simpler framework to obtain such representation theorems.
\end{abstract}

\section{Introduction}

A consequence relation $\sim$ is a binary relation between formulas on a classical propositional language. We are interested in nonmonotonic consequence relations, i.e. those relations that do not satisfy the monotonicity rule: If $\alpha \uparrow \gamma$ then $\alpha \wedge \beta \uparrow \gamma$. Several systems of postulates (cumulative, preferential, rational and others) for classifying nonmonotonic consequence relations has been investigated $[6$, $7,4,3,2,5]$. One of the main features of these systems is the amount of monotony that is required from the consequence relation. The study of non monotonic reasoning has been motivated by problems arising in artificial intelligence (knowledge representation, belief revision, etc). There is a vast literature concerning nonmonotonity, for the particular approach dealt with in this paper we refer the reader to $[9,6]$ and the references therein.

An important tool for the study and classification of nonmonotonic consequence relations is the representation of such relations in terms of preferential models. A preferential model $\mathcal{M}$ is a triple $\langle S, \imath, \prec\rangle$, where $S$ is a set of states, $\imath$ is function assigning to each state a valuation and $\prec$ is a binary relation over $S . \mathcal{M}$ is said to be a model of $\sim$ when $\alpha \sim \beta$ iff $\imath(s) \models \beta$ for all $s$ which are $\prec$-minimal among all states $t$ such that $\imath(t) \models \alpha$ (the details are given in $\S 2$ ). A consequence relation $\sim$ is preferential relation $\sim$ if and only if it is of the form $\sim_{\mathcal{M}}$ for some preferential model $\mathcal{M}$ ([6]). If $\sim$ is rational then the model can be found ranked ([7]). Disjunctive relations were studied in [2] and shown to be those relations represented by filtered models. When the relation also satisfies rational transitivity then $\mathcal{M}$ can be found quasi-linear $([1,5])$. These results are referred to as representation theorems and they can be regarded as a sort of a soundness and completeness theorems. These representations, besides providing a semantic interpretation of $\sim$, are also quite useful to establish most properties of $\sim$ by model theoretic arguments instead of proof theoretic ones.

In this paper we give simpler proofs of all representation theorems for injective relations mentioned above. The key idea is the notion of the essential relation $<_{e}$ (defined in $\S 3$ ) associated with a preferential consequence relation $\sim$. We will show that if $\sim$ is preferential and disjunctive, then $<_{e}$ is a transitive strict order defined on a set of valuations such that the models of $\{\beta: \alpha \sim \beta\}$ are the $<_{e}$-minimal valuations that satisfy $\alpha$. In other words, $<_{e}$ provides a representation of $\sim$. We 
will show also that if $\sim$ is disjunctive (resp. rational, rational transitive), then $<_{e}$ is filtered (resp. ranked, quasi-linear). Most of these results were known but they were proved by quite different means (see $[6,7,2,4,5]$ ). We think our proofs are easier and in a sense "canonical". One interesting feature of our approach is that $<_{e}$ provides a direct way of "ordering" the valuations without using an auxiliary order over formulas, as is the case of other proofs of representation theorems. Freund introduced a property (that we denote by WDR) weaker than disjunctiveness. We show that if $\sim$ is preferential and satisfies WDR, then $<_{e}$ represents $\sim$. We will see in $\S 4$ that in spite of the fact that in some cases $<_{e}$ is not transitive, it still provides a good representation of some preferential relations for which other methods do not work.

A preliminary version of this work appears as a technical report N IT-300, Universite de Lille I. April 1997.

\section{Preliminaries}

We recall some basic definitions and results from Kraus, Lehmann and Magidor [6], Lehmann and Magidor [7] and Freund [2] which will be used in the paper.

We consider formulas of classical propositional calculus built over a set of variables denoted Var plus two constants $T$ and $\perp$ (the formulas true and false respectively). Let $\mathcal{L}$ be the set of formulas. If $\operatorname{Var}$ is finite we will say that the language $\mathcal{L}$ is finite. Let $\mathcal{U}$ be the set of valuations (or worlds), i.e. functions $M: \operatorname{Var} \cup\{\top, \perp\} \longrightarrow\{0,1\}$ such that $M(T)=1$ and $M(\perp)=0$. We use lower case letters of the Greek alphabet to denote formulas, and the letters $M, N, P, M_{1}, M_{2}, \ldots$ to denote worlds. As usual, $\vdash \alpha$ means that $\alpha$ is a tautology and $M \models \alpha$ means that $M$ satisfies $\alpha$ where compound formulas are evaluated using the usual truth-functional rules. We consider certain binary relations between formulas. These relations will be called consequence relations and will be written $\sim$.

Definition 2.1 $A$ relation $\sim$ is said to be cumulative iff the following rules hold

$\begin{array}{ll}\text { REF } & \forall \alpha[\alpha \sim \alpha] \\ \text { LLE } & \forall \alpha, \beta, \gamma[\alpha \sim \beta \& \vdash \alpha \leftrightarrow \gamma \Rightarrow \gamma \vdash \beta] \\ \text { RW } & \forall \alpha, \beta, \gamma[\alpha \vdash \beta \& \vdash \beta \rightarrow \gamma \Rightarrow \alpha \sim \gamma] \\ \text { CUT } & \forall \alpha, \beta, \gamma[\alpha \wedge \beta \vdash \gamma \& \alpha \sim \beta \Rightarrow \alpha \sim \gamma] \\ \text { CM } & \forall \alpha, \beta, \gamma[\alpha \sim \beta \& \alpha \sim \gamma \Rightarrow \alpha \wedge \gamma \vdash \beta]\end{array}$

These rules are known as the rules of the system $C$. The abbreviations above are read as follows: REF -reflexivity, LLE -left logical equivalence, RW -right weakening, CM -cautious monotony. CUT is self-explanatory, but it should be noted that this form of cut, which plays an important role in nonmonotonic logic, is weaker than the form of cut usually studied in Gentzen-style formulations of classical and intuitionistic logic. The latter implies transitivity of the inference relation; the former does not.

It is well known [6] that the following rules (And, Reciprocity) are derivable from system C:

$\begin{array}{ll}\text { AND } & \forall \alpha, \beta, \gamma[\alpha \sim \beta \& \alpha \sim \gamma \Rightarrow \alpha \sim \beta \wedge \gamma] \\ \text { RECIP } & \forall \alpha, \beta, \gamma[\alpha \sim \beta \& \beta \sim \alpha \& \alpha \sim \gamma \Rightarrow \beta \sim \gamma]\end{array}$


Definition 2.2 A relation $\sim$ is said to be preferential iff it is cumulative and satisfies the following rule (or):

$$
\text { OR } \forall \alpha, \beta, \gamma[\alpha \vdash \gamma \& \beta \vdash \gamma \Rightarrow \alpha \vee \beta \vdash \gamma]
$$

A relation $\sim$ is said to be disjunctive rational iff it is preferential and the following rule (disjunctive rationality) holds

$$
\text { DR } \quad \forall \alpha, \beta, \gamma[\alpha \vee \beta \sim \gamma \& \alpha \not<\gamma \Rightarrow \beta \sim \gamma]
$$

$A$ relation $\sim$ is said to be rational iff it is preferential and the following rule (rational monotony) holds

$$
\text { RM } \quad \forall \alpha, \beta, \gamma[\alpha \sim \beta \& \alpha \not \chi \neg \gamma \Rightarrow \alpha \wedge \gamma \vdash \beta]
$$

It is well known $[6,9]$ that given the preferential rules (system $C$ plus OR), RM implies DR and also that any preferential relation satisfies the following rule

$$
\text { S } \quad \forall \alpha, \beta, \gamma[\alpha \wedge \beta \sim \gamma \Rightarrow \alpha \sim \beta \rightarrow \gamma]
$$

Let $\sim$ be a consequence relation. As usual, $C_{\sim}(\alpha)=\{\beta: \alpha \sim \beta\}$. If there is no ambiguity we shall write $C(\alpha)$ instead of $C_{\mathrm{r}}(\alpha)$. If $U(\alpha)$ is a set of formulas (a formula) then $C n(U)(C n(\alpha)$ ) will denote the set of classical consequences of $U(\alpha)$.

We recall the definition of preferential models.

Definition 2.3 A structure $\mathcal{M}$ is a triple $\langle S, \imath, \prec\rangle$ where $S$ is a set (called the set of states), $\prec$ is a strict order (i.e. transitive and irreflexive) on $S$ and $\imath: S \longrightarrow \mathcal{U}$ is a function (called the interpretation function).

Let $\mathcal{M}=\langle S, \imath, \prec\rangle$ be a structure. We adopt the following notations: if $T \subseteq S$, then $\min (T)=$ $\left\{t \in T: \neg \exists t^{\prime} \in T, t^{\prime} \prec t\right\}$, i.e. $\min (T)$ is the set of all minimal elements of $T$ with respect to $\prec$; $\bmod _{\mathcal{M}}(\alpha)=\{s \in S: \imath(s) \models \alpha\} ; \min _{\mathcal{M}}(\alpha)=\min \left(\bmod _{\mathcal{M}}(\alpha)\right)$.

Definition 2.4 Let $\mathcal{M}=\langle S, \imath, \prec\rangle$ be a structure and $T \subseteq S$. We say that $T$ is smooth if it satisfies the following

$$
\forall s \in T \backslash \min (T) \quad \exists s^{\prime} \in \min (T) s^{\prime} \prec s
$$

$\mathcal{M}$ is said to be a preferential model if $\bmod _{\mathcal{M}}(\alpha)$ is smooth for any formula $\alpha$.

Each preferential model has associated a consequence relation given by the following:

Definition 2.5 Let $\mathcal{M}=\langle S, \imath, \prec\rangle$ be a preferential model. The inference relation $\sim_{\mathcal{M}}$ is defined by the following

$$
\alpha \sim_{\mathcal{M}} \beta \Leftrightarrow \min _{\mathcal{M}}(\alpha) \subseteq \bmod _{\mathcal{M}}(\beta)
$$

The following representation theorems are one of the basic tools in the study of nonmonotonic consequence relations. The if part of them are not difficult to establish. The main subject of this paper consists in providing, for a large class of preferential relations, a 'canonical' way of proving the only if part. 
Theorem 2.6 (Krauss, Lehmann and Magidor [6]) A consequence relation $\downarrow$ is preferential iff there is a preferential model $\mathcal{M}$ such that $\sim=\sim_{\mathcal{M}}$.

A structure $\mathcal{M}=\langle S, \imath, \prec\rangle$ is said to be a ranked model if it is a preferential model and there exists a strict linear order $(\Omega,<)$ and a function $r: S \longrightarrow \Omega$ such that for any $s, s^{\prime} \in S, s \prec s^{\prime}$ iff $r(s)<r\left(s^{\prime}\right)$.

Theorem 2.7 (Lehmann and Magidor [7]) A consequence relation $\sim$ is rational iff there is a ranked model $\mathcal{M}$ such that $\sim=\sim_{\mathcal{M}}$.

In general, it is not easy to grasp the intuition behind the set of states $S$ and the interpretation function $\imath$. A particular case, which is intuitively easy to handle, is when the function $\imath$ is injective (in this case, $\mathcal{M}$ is said to be an injective model). If a preferential model is injective one does not need to mention the interpretation function $\imath$, instead one can assume that $S$ is a set of valuations and $\prec$ is a strict smooth order over $S$, so $\imath$ would be the identity function. In this case the notion of a smooth relation says that for every $M \in S$ and for every formula $\alpha$ if $M \models \alpha$ and $M$ is not in $\min (\bmod (\alpha) \cap S, \prec)$, then there is $N \in S$ such that $N \prec M$ and $N \in \min (\bmod (\alpha) \cap S, \prec)$ (where the notion of a $\prec$-minimal element is defined as in the paragraph following 2.3). The relation $\prec$ is understood as a preference relation over valuations. Thus (1) says that to compute the consequences of a formula $\alpha$ we need to look only at the preferred valuations of $\alpha$ according to $\prec$, i.e. those valuations belonging to $\min (\bmod (\alpha) \cap S)$.

Freund [2] studied some consequence relations admitting injective models. He observed that one can always assume that $S$ is certain collection of valuations which we defined next

Definition 2.8 Let $\sim$ be a consequence relation. A valuation $N$ is called normal w.r.t. $\sim$ if there is a formula $\alpha$ such that $N \vDash C(\alpha)$.

If there is not ambiguity we shall say that an interpretation is normal instead of normal with respect to $\mathcal{W}$ (in [2] normal valuations were called $\sim$-consistent). Freund showed (see remark 3.1 in [2]) that if $\sim$ is represented by an injective model then it can also be represented by an injective model where the set $S$ is the collection of all normal valuation w.r.t. $\sim$. Taking all this considerations into account we introduce the following

Definition 2.9 Let $\sim$ be a consequence relation and $S$ the collection of normal valuation w.r.t $\sim$. We say that $\sim$ is represented by an injective model if there is a smooth strict order $\prec$ over $S$ such that

$$
\alpha \sim \beta \Leftrightarrow \min (\bmod (\alpha) \cap S, \prec) \subseteq \bmod (\beta)
$$

Let us observe that $(2)$ can be restated in the following way: $\min (\bmod (\alpha) \cap S,<) \subseteq \bmod (C(\alpha))$. Some consequence relations admit an injective representation where the equality holds. They were called standard in [2], the formal definition is the following

Definition 2.10 Let $\sim$ be a consequence relation and $S$ the collection of normal valuation w.r.t $\sim$. We say that $\sim$ is represented by a standard model if there is a smooth strict order $\prec$ over $S$ such that

$$
\bmod (C(\alpha))=\min (\bmod (\alpha) \cap S, \prec)
$$

Such order $\prec$ will be called a standard order that represents $\uparrow$. 


\section{The essential relation and standard representations}

It is not difficult to show that if the language is finite the notions of an injective and a standard models coincide (see [2]) but this is not the case if the language is infinite (an example will be given in $\$ 4)$. Freund characterized some preferential relations that admit a standard representation. In the case of a finite language his characterization is quite easy to state. The following property is called Weak Disjunctive Rationality

$$
\text { WDR } C(\alpha \vee \beta) \subseteq C n(C(\alpha) \cup C(\beta))
$$

Freund showed that for a finite language, a preferential relation admits an injective (thus standard) model iff it satisfies WDR. However, for infinite languages, he introduced a property stronger than WDR which is based in the notion of a trace of a formula. The aim of this section is to give simple and uniform proofs of representation theorems for consequence relations that satisfy WDR.

We define next the essential relation, which will play a key role in this paper. We will show that this relation can be considered the canonical relation that represents a given preferential consequence relation that satisfies WDR. The essential relation seems easier to handle than the relation defined by Freund. We will see in $\S 4$ that they are equal under some conditions. However, we will also give an example of a preferential relation $\sim$ represented by our relation but not by Freund's. The idea behind the definition of the essential relation seems to be quite general and turns out to be also useful in a different context (see [10]).

Notation: Given a consequence relation $\sim$ we will always denote by $S(\sim)$ the collection of normal valuation w.r.t $\sim$, when there is no ambiguity about which consequence relation is used we will just write $S$. If $M$ is a valuation, $T h(M)$ will denote the theory of $M$, i.e. $T h(M)=\{\alpha: M \models \alpha\}$. For a fixed inference relation $\sim$ and a valuation $M, T^{\sim}(M)$ will denote the set $\{\alpha: M \models C(\alpha)\}$, i.e. a sort of "nonmonotonic theory" of $M$. If there is no ambiguity we write $T(M)$ instead of $T^{\sim}(M)$.

Definition 3.1 Let $\sim$ be a consequence relation. The essential relation is defined by the following: Let $N$ and $M$ normal valuations,

$$
M<_{e} N \Longleftrightarrow \forall \alpha(N \models C(\alpha) \Rightarrow M \not \alpha)
$$

In other words, $M<_{e} N$ iff $T h(M) \cap T(N)=\emptyset$.

The essential relation is not in general transitive (we will see an example in $\S 4$ ). It is not difficult to show that transitivity of $\prec$ is not necessary in order to get the easy half of 2.6 (but smoothness can not be avoided). This was already observed in [6]) and we state it for later reference.

Lemma 3.2 ([6]) Let < be a binary non-reflexive (but not necessarily transitive) smooth relation over a set $T$ of valuations. Define $\sim$ by $\alpha \sim \beta$ iff $\min (\bmod (\alpha) \cap T,<) \subseteq \bmod (\beta)$. Then $\sim$ is preferential.

We will see that under the presence of WDR the relation $<_{e}$ is smooth and still represents $\sim$ in the sense that equation in 2.10 holds. For this reason we will use the following notion, which is more permissive than that of a standard model. 
Definition 3.3 Let $\sim$ a consequence relation and $\prec$ a binary relation over $S$. We say that $\prec$ is a standard relation that represents $\sim$ if the following holds

$$
\bmod (C(\alpha))=\min (\bmod (\alpha) \cap S, \prec)
$$

We emphasize that we do not ask the relation to be a strict smooth order, but in most interesting cases the relation will be smooth. We show next that (3) implies that $\sim$ satisfies WDR.

Lemma 3.4 Suppose $\sim$ is a consequence relation and $<$ is a standard relation that represents $\sim$. Then $\sim$ satisfies WDR.

Proof: Let $N \models C(\alpha) \cup C(\beta)$, we have to show that $N \models C(\alpha \vee \beta)$. From (3) we have that $N \in \min (\bmod (\alpha)) \cap \min (\bmod (\beta))$. It is easy to check that $N \in \min (\bmod (\alpha \vee \beta))$.

The following observation shows that the essential relation associated with $\sim$ is finer than any standard relation representing $\sim$.

Lemma 3.5 Let $\sim$ be a consequence relation and $<$ a standard relation that represents $\uparrow$. Then for all normal valuation $N$ and $M$, if $N<M$, then $N<_{e} M$.

Proof: Suppose $N$ and $M$ are normal valuation such that $N \Varangle_{e} M$. That is to say, there is $\alpha$ such that $N \models \alpha$ and $M \models C(\alpha)$. By hypothesis $M \in \min (\bmod (\alpha) \cap S,<)$, therefore $N \nless M$.

The following observation is obvious and says that $<_{e}$ satisfies one half of (3) without any hypothesis about $\sim$.

Lemma 3.6 Let $\sim$ be a consequence relation. If $M \models C(\alpha)$ then $M \in \min \left(\bmod (\alpha) \cap S,<_{e}\right)$.

The following observation is well known [9]

Lemma 3.7 Let $\sim$ be a cumulative relation. If $\alpha \uparrow \beta$ then $C(\alpha)=C(\alpha \wedge \beta)$.

Lemma 3.8 Let $\sim$ be a preferential relation. If $M \models \alpha$ and $M \models C(\beta)$ then $M \models C(\alpha \wedge \beta)$.

Proof: Suppose $\alpha \wedge \beta \vdash \gamma$. We want to show that $M \models \gamma$. By the $\mathrm{S}$ rule, $\beta \sim \alpha \rightarrow \gamma$, since $M \models C(\beta)$ then $M \models \alpha \rightarrow \gamma$. Since $M \models \alpha$, then $M \models \gamma$.

Since we are dealing with non monotonic consequence relations we can not expect the set $T(M)$ to be closed under $\wedge$ (not even in the case of a rational consequence relation). On the other hand, in general, $T(M)$ is not closed under $\vee$. The next lemma establish under which condition $T(M)$ is closed under $\vee$.

Lemma 3.9 $\sim$ satisfies WDR if and only if for any $M, T(M)$ is closed under the connective $v$, i.e. for any $\beta_{1}, \beta_{2} \in T(M),\left(\beta_{1} \vee \beta_{2}\right) \in T(M)$. 
Proof: Suppose that $\beta_{1}, \beta_{2} \in T(M)$, so $M \vDash C\left(\beta_{i}\right)$ for $i=1,2$. Thus $M \vDash C n\left(C\left(\beta_{1}\right) \cup C\left(\beta_{2}\right)\right)$. By WDR, $C\left(\beta_{1} \vee \beta_{2}\right) \subseteq C n\left(C\left(\beta_{1}\right) \cup C\left(\beta_{2}\right)\right)$, then we have $M \models C\left(\beta_{1} \vee \beta_{2}\right)$, i.e. $\left(\beta_{1} \vee \beta_{2}\right) \in T(M)$. The other direction is also straightforward.

The following result is the basic representation theorem in this paper. All others representation theorems that we will show are based on it and will only add that $<_{e}$ has nicer properties (like being transitive, filtered, modular or quasi-linear) when the preferential relation $\sim$ satisfies some extra postulates besides WDR. This theorem is a generalization of Freund's main representation theorem (see his theorem 4.11 in [2]).

Theorem 3.10 Let $\sim$ be a consequence relation. Then $\sim$ is a preferential relation satisfying WDR if, and only if $<_{e}$ is a smooth standard relation representing $\sim$.

Proof: The if part follows from 3.2 and 3.4. For the only if part we start by showing that $<_{e}$ is irreflexive. If $M$ is normal then there exists $\alpha$ such that $M \models C(\alpha)$, so $T h(M) \cap T(M) \supseteq\{\alpha\} \neq \emptyset$, i.e. $M \nless_{e} M$.

Now we show that $<_{e}$ is smooth. Let $M \in \bmod (\alpha) \cap S$. We want to show that either $M \in \min \left(\bmod (\alpha) \cap S,<_{e}\right)$ or there exists $N \in \min \left(\bmod (\alpha) \cap S,<_{e}\right)$ with $N<_{e} M$. We consider two cases: $M \vDash C(\alpha)$ or $M \not \models C(\alpha)$. In the former case, by lemma 3.6, we have $M \in$ $\min \left(\bmod (\alpha) \cap S,<_{e}\right)$. In the latter case define $U=C(\alpha) \cup\{\neg \beta: \beta \in T(M)\}$. We claim that $U$ is consistent. Otherwise by compactness there are $\alpha_{1}, \ldots, \alpha_{m}$ in $C(\alpha)$ and $\beta_{1}, \ldots, \beta_{n}$ in $T(M)$ such that $\left\{\alpha_{1}, \ldots, \alpha_{m}, \neg \beta_{1}, \ldots, \neg \beta_{n}\right\} \vdash \perp$. Hence $\alpha_{1} \wedge \cdots \wedge \alpha_{m} \vdash \beta_{1} \vee \cdots \vee \beta_{n}$. Put $\beta=\beta_{1} \vee \cdots \vee \beta_{n}$. By AND, $\alpha \sim \alpha_{1} \wedge \cdots \wedge \alpha_{m}$, so by RW, $\alpha \sim \beta$. Hence, by lemma 3.7, $C(\alpha)=C(\alpha \wedge \beta)$. By lemma 3.9 , $\beta \in T(M)$. Thus by lemma 3.8, $M \models C(\alpha \wedge \beta)$, i.e. $M \models C(\alpha)$, a contradiction. Now consider $N$ such that $N \vDash U$. By definition of $U, N \models C(\alpha)$ so by lemma 3.6, $N \in \min \left(\bmod (\alpha) \cap S,<_{e}\right)$. Also by definition of $U$ it is clear that $N<_{e} M$.

To see that $<_{e}$ is a standard relation that represents $\sim$ it suffices to show that if $M \in$ $\min \left(\bmod (\alpha) \cap S,<_{e}\right)$ then $M \vDash C(\alpha)$, the other direction is given by 3.6. But this was already shown above, since we have proved that if $M \not \models C(\alpha)$, then $M \notin \min \left(\bmod (\alpha) \cap S,<_{e}\right)$.

Since the usual definition of a standard model requires transitivity of the relation, it is quite natural to ask when is $<_{e}$ transitive. We will see in $\S 4$ an example of a preferential relation satisfying WDR for which $<_{e}$ is not transitive. The next lemma says that for the case of a finite language it suffices that $\sim$ satisfies WDR. Later we will see that, in general, if $\sim$ is disjunctive, then $<_{e}$ is transitive.

Lemma 3.11 Suppose that the language is finite and $\vdash$ is preferential and satisfies WDR, then $<_{e}$ is transitive.

Proof: Suppose that $M<_{e} N$ and $N<_{e} P$; we want to show that $M<_{e} P$. Since the language is finite for every valuation $Q$ there is a formula $\gamma_{Q}$ such that $\bmod \left(\gamma_{Q}\right)=\{Q\}$. Now consider the formula $\alpha=\gamma_{M} \vee \gamma_{N} \vee \gamma_{P}$. Note that $\bmod (\alpha)=\{M, N, P\}$. By the assumptions, $M$ is the only element of $\{M, N, P\}$ which can be minimal in $\bmod (\alpha)$. Therefore by the smoothness of $\bmod (\alpha)$, $M<_{e} P$. 
Putting together $3.2,3.4,3.10$ and 3.11 we obtain the following result which is essentially the same result of Freund (see his theorem 4.13 in [2]) but with a different proof.

Theorem 3.12 Assume the language is finite. Then $\sim$ is a preferential relation satisfying WDR if and only if $<_{e}$ is a standard order that represents $\sim$.

Now we will look at stronger consequence relations. We start with relation that satisfies disjunctive rationality DR. Next remark is trivial but useful

Lemma 3.13 DR is equivalent to saying that $C(\alpha \vee \beta) \subseteq C(\alpha) \cup C(\beta)$ for all formulas $\alpha$ and $\beta$. In particular, any consequence relation satisfying DR satisfies WDR.

Lemma 3.14 The following properties are equivalent for a cumulative relation $\vdash$ :

(i) The relation $\sim$ satisfies $\mathrm{DR}$.

(ii) For any valuations $M, N$ and for any formulas $\alpha, \beta$ if $M \models C(\alpha)$ and $N \models C(\beta)$ then either $M \vDash C(\alpha \vee \beta)$ or $N \vDash C(\alpha \vee \beta)$.

Proof: $\quad(i \Rightarrow i i)$ Suppose $M \models C(\alpha)$ and $N \vDash C(\beta)$. For reductio, suppose $M \not \models C(\alpha \vee \beta)$ and $N \forall \forall C(\alpha \vee \beta)$. Then there are formulas $\gamma_{1}, \gamma_{2} \in C(\alpha \vee \beta)$ such that $M \not \forall \gamma_{1}$ and $N \forall \forall \gamma_{2}$. By AND, $\gamma_{1} \wedge \gamma_{2} \in C(\alpha \vee \beta)$, so by $3.13 \gamma_{1} \wedge \gamma_{2} \in C(\alpha)$ or $\gamma_{1} \wedge \gamma_{2} \in C(\beta)$. But in both cases we get a contradiction because neither $M$ nor $N$ are models of $\gamma_{1} \wedge \gamma_{2}$.

(ii $\Rightarrow$ i) Suppose $\gamma \in C(\alpha \vee \beta)$. We want to show that $\gamma \in C(\alpha)$ or $\gamma \in C(\beta)$. Suppose not. Then there are valuations $M, N$ such that $M \models C(\alpha), N \models C(\beta), M \not \gamma$ and $N \not \neq \gamma$. By (ii), $M \vDash C(\alpha \vee \beta)$ or $N \vDash C(\alpha \vee \beta)$. But in both cases we get a contradiction because neither $M$ nor $N$ are models of $\gamma$.

The following relation between valuations was defined in [8]. We came up with the definition of $<_{e}$ by trying to extend the results in [8] to the case of an infinite language and to a larger class of consequence relations.

Definition 3.15 Let $\sim$ a consequence relation. We define the relation $<_{u}$ over the normal valuations by:

$$
M<{ }_{u} N \Longleftrightarrow \forall \alpha \forall \beta(M \models C(\alpha) \text { and } N \vDash C(\beta) \Rightarrow M \models C(\alpha \vee \beta) \text { and } N \not \models C(\alpha \vee \beta))
$$

The relation $<_{u}$ is quite more intuitive and we show next that it is equal to $<_{e}$ under the presence of DR.

Lemma 3.16 Let $\sim$ a disjunctive rational relation. Then $<_{e}$ is equal to $<_{u}$. 
Proof: $\left(<_{e} \subseteq<_{u}\right)$ Suppose $M<_{e} N, M \models C(\alpha), N \models C(\beta)$. We want to show that $M \models C(\alpha \vee \beta)$ and $N \not \models C(\alpha \vee \beta)$. Since $M \models \alpha, M \models \alpha \vee \beta$ and $M<_{e} N$, then $N \not \models C(\alpha \vee \beta)$. Therefore by proposition $3.14, M \models C(\alpha \vee \beta)$.

$\left(<_{u} \subseteq<_{e}\right)$ Suppose $M<_{u} N$. We want to show that $T h(M) \cap T(N)=\emptyset$. Suppose not, then there is a formula $\beta$ such that $M \models \beta$ and $N \models C(\beta)$. Let $\alpha$ be a formula such that $M \models C(\alpha)$. By lemma 3.8, $M \models C(\alpha \wedge \beta)$; and since $M<_{u} N$, then $N \not \models C((\alpha \wedge \beta) \vee \beta)$. But $\vdash((\alpha \wedge \beta) \vee \beta) \leftrightarrow \beta$, so $N \not \forall C(\beta)$, a contradiction.

Lemma 3.17 If the relation $\sim$ is disjunctive rational then $<_{e}$ is transitive.

Proof: Suppose $N<_{e} M$ and $M<_{e} P$ but $N \nless_{e} P$. Let $\alpha$ be such that $P \models C(\alpha)$ and $N \models \alpha$. Let $\beta$ be such that $M \models C(\beta)$, then it follows from the definition of $<_{e}$ that $P \not \subset C(\alpha \vee \beta)$ and $M \not \models C(\alpha \vee \beta)$. By $3.14 \sim$ does not satisfy DR.

The following definition is due to Freund [2]

Definition 3.18 An order $\prec$ over valuations is filtered iff for any formula $\alpha$ and any valuations $M, N \in \bmod (\alpha)$ such that $M \notin \min (\alpha)$ and $N \notin \min (\alpha)$ there exists $P \in \min (\alpha)$ such that $P \prec M$ and $P \prec N$.

Lemma 3.19 If $\sim$ is disjunctive rational then $<_{e}$ is filtered.

Proof: The argument is very close to that in the proof of the smoothness of $<_{e}$ ( $c f$. proof of proposition 3.10). By hypothesis and lemma 3.6, $M \not \forall C(\alpha)$ and $N \not \neq C(\alpha)$. Put $U=$ $C(\alpha) \cup\{\neg \beta: \beta \in T(M)\} \cup\{\neg \gamma: \gamma \in T(N)\}$. We claim that $U$ is consistent. Suppose not, then by compactness there are $\alpha_{1}, \ldots, \alpha_{m}$ in $C(\alpha), \beta_{1}, \ldots, \beta_{n}$ in $T(M)$ and $\gamma_{1}, \ldots, \gamma_{r}$ in $T(N)$ such that $\left\{\alpha_{1}, \ldots, \alpha_{m}, \neg \beta_{1}, \ldots, \neg \beta_{n}, \neg \gamma_{1}, \ldots, \neg \gamma_{r}\right\} \vdash \perp$. Hence $\alpha_{1} \wedge \cdots \wedge \alpha_{m} \vdash \beta_{1} \vee \cdots \vee \beta_{n} \vee \gamma_{1} \vee \cdots \vee \gamma_{r}$. Put $\beta=\beta_{1} \vee \cdots \vee \beta_{n}$ and $\gamma=\gamma_{1} \vee \cdots \vee \gamma_{r}$. By AND, $\alpha \sim \alpha_{1} \wedge \cdots \wedge \alpha_{m}$ and by RW, $\alpha \sim \beta \vee \gamma$. By observation $3.9, \beta \in T(M)$ and $\gamma \in T(N)$. Thus by proposition $3.14 M \models C(\beta \vee \gamma)$ or $N \vDash C(\beta \vee \gamma)$. Without lost of generality suppose that $M \models C(\beta \vee \gamma)$ (the other case is similar). By lemma 3.8, $M \models C(\alpha \wedge(\beta \vee \gamma))$ and since $\alpha \sim \beta \vee \gamma$, then by lemma 3.7, $C(\alpha)=C(\alpha \wedge(\beta \vee \gamma))$, hence $M \models C(\alpha)$, a contradiction. Hence $U$ is consistent. Let $P$ be a model of $U$. By definition of $U, P \models C(\alpha)$, $P<_{e} M$ and $P<_{e} N$. So by $3.6 P \in \min (\alpha)$

Freund [2] has shown that a consequence relation is disjunctive rational if and only if it has a standard filtered model. The next theorem is the hard half of his result with a different proof. The theorem follows from $3.10,3.17$, and 3.19 .

Theorem 3.20 Let $\sim$ be a disjunctive rational relation. Then $<_{e}$ is a standard filtered order representing $\vdash$.

Now we look at the properties that $<_{e}$ would have in the presence of rational monotony RM. It is not difficult to check the well known fact (see [6]) that any rational relation satisfies DR. Thus, 
if $\mathcal{W}$ is rational then $<_{e}$ is filtered and in particular. transitive. We have already mentioned that rational relations are represented by ranked models (see 2.7). A preferential model is ranked when the order relation is modular. We recall the definition of modular relation (see [7]):

Definition 3.21 A relation $<$ on $E$ is said to be modular iff there exists a strict linear order $\prec$ on some set $\Omega$ and a function $r: E \longrightarrow \Omega$ such that $a<b \Leftrightarrow r(a) \prec r(b)$.

The following characterization of modularity is well-known and easy to verify.

Lemma 3.22 An order $<$ on $E$ is modular iff for any $a, b, c \in E$ if $a$ and $b$ are incomparable and $a<c$ then $b<c$.

The following result is well known and we include its proof for the sake of completeness.

Lemma 3.23 Let $\sim$ be a rational relation. If $\alpha \nmid \neg \beta$, then $C(\alpha \wedge \beta)=C n(C(\alpha) \cup\{\beta\})$

Proof: Let $\delta \in C(\alpha)$ then by RM we have $\delta \in C(\alpha \wedge \beta)$. Thus $C n(C(\alpha) \cup\{\beta\}) \subseteq C(\alpha \wedge \beta)$. For the other inclusion, if $\alpha \wedge \beta \sim \delta$ then by the rule $\mathrm{S}$ we have $\alpha \sim \beta \rightarrow \delta$. Therefore $\delta \in C n(C(\alpha) \cup\{\beta\})$.

The next result shows that under the presence of RM it is quite easy to check that $N<_{e} M$.

Lemma 3.24 Let $\uparrow$ be a rational relation and $N, M$ be normal models. Then $N<_{e} M$ if and only if there are $\alpha$ and $\beta$ formulas such that $N \models C(\alpha), M \models C(\beta)$ and $N \models C(\alpha \vee \beta)$ but $M \not \models C(\alpha \vee \beta)$.

Proof: The only if part comes from 3.16 (recall that rational relations are in particular disjunctive rational). For the if part, suppose that such $\alpha$ and $\beta$ exist, we will show that $N<_{u} M$. Let $\gamma$ and $\delta$ be any formulas such that $N \models C(\gamma)$ and $M \models C(\delta)$. From proposition 3.14 we get that $\gamma \vee \delta \not \neg \neg(\alpha \vee \beta)$ and also $\alpha \vee \beta \not \neg \neg(\gamma \vee \delta)$. Hence from lemma 3.23 we get that

$$
\begin{aligned}
C((\alpha \vee \beta) \wedge(\gamma \vee \delta)) & =C n(C(\gamma \vee \delta) \cup\{\alpha \vee \beta\}) \\
& =C n(C(\alpha \vee \beta) \cup\{\gamma \vee \delta\})
\end{aligned}
$$

and from this the result follows because $N \models C n(C(\alpha \vee \beta) \cup\{\gamma \vee \delta\})$ so $N \models C(\gamma \vee \delta)$ and since $M \not \models C n(C(\alpha \vee \beta) \cup\{\gamma \vee \delta\})$ and $M \models \alpha \vee \beta$, we have $M \not \forall C(\gamma \vee \delta)$.

A straightforward consequence of this lemma is the following

Lemma 3.25 Let $\sim$ be a rational relation and $N, M$ be normal models. $N \nless_{e} M$ and $M \nless_{e} N$ if and only if $N, M \models C(\gamma \vee \delta)$ for all formulas $\gamma$ and $\delta$ such that $N \models C(\gamma), M \models C(\delta)$.

Lemma 3.26 If the relation $\sim$ is rational then $<_{e}$ is modular. 
Proof: Let $M, N, P$ be normal valuations. Suppose $N \nless_{e} M, M \nless_{e} N$ and $M<_{e} P$. By 3.22 it suffices to show that $N<_{e} P$. Let $\alpha, \beta, \gamma$ be formulas such that $M \models C(\alpha), N \models C(\beta)$ and $P \models C(\gamma)$. Since $M$ and $N$ are incomparable, by lemma 3.25 we have $M \models C(\alpha \vee \beta)$ and $N \models C(\alpha \vee \beta)$. We claim that $P \forall C(\alpha \vee \beta \vee \gamma)$ and $N \models C(\alpha \vee \beta \vee \gamma)$, which implies, by lemma 3.24 , that $N<_{e} P$. To prove the claim it suffices (by lemma 3.14) to see that $P \not \models C(\alpha \vee \beta \vee \gamma)$. Since $M<_{e} P$ and $M \models C(\alpha \vee \beta)$ and $P \models C(\gamma)$, then $P \not C C(\alpha \vee \beta \vee \gamma)$.

Now putting together 3.20 and 3.26 we get the following well known theorem which has been proved in many different ways $([7,4,2])$. We will see in $\S 4$, that $<_{e}$ is in fact the unique standard modular order that represents a given rational relation.

Theorem 3.27 If $\sim$ is a rational relation then $<_{e}$ is a standard and modular relation that represents $\sim$.

To finish this section we will comment about a postulate stronger than rational monotony. A relation $\sim$ is rational transitive, if it is preferential and the following rule (RT) holds

$$
\text { RT } \frac{\alpha \vdash \beta \quad \beta \sim \gamma \quad \alpha \not \not \neg \gamma}{\alpha \sim \gamma}
$$

It is known that rational transitive consequence relations satisfies RM and that rational transitive consequence relations are represented by 'quasi-linear' standard relations (a relation < is quasilinear if $M$ is a valuation that is not minimal then for any valuation $N$ different of $M$ we have $N<M$ or $M<N$ ) (see [1, 5]). If $\sim$ is rational transitive then $<_{e}$ is quasi-linear (this follows from proposition 5.6 of [5]).

\section{Further properties of $<_{e}$ and some comments about standard models.}

In this section we address the problem of when a consequence relation has a unique representation. Let us make first some simple observations to put the question in the right setting. Our definition of an injective model for a consequence relation $\sim$ (see definition 2.9) requires that the order relation $\prec$ has to be on the collection $S$ of all normal valuations w.r.t. $\sim$. We observed (just before introducing definition 2.9) that this requirement can be assumed without any lost of generality. In other words, there are consequence relations $\sim$ that can be represented (as in 2.5) by various order relations defined on different sets of valuations. But there is always at least one such relation defined on the entire set $S$. It is nothing strange that there are so many representations, just recall that only countable many valuations are needed to define the semantic counterpart $\models$ of the classical entailment relation $\vdash$. Taking this considerations into account, the question we want to address is whether for a given preferential relation $\sim$ (admitting an injective model) there is a unique order on $S$ representing $\sim$. In this generality, this uniqueness seems to be quite rare when the language is infinite (it holds when it is finite). So we will also address a more restrictive question: if there is a standard model, when is it unique? 
It is well known that a subset $T$ of the collection of valuations $\mathcal{U}$ suffices to define the classical relation $\models$ iff $T$ is topologically dense in $\mathcal{U}$ with respect to a natural topology associated with $\mathcal{U}$. This topology turns out to be quite useful in relation with the problems we address in this section. Its use will make some proofs short and simple, and more important, we will show that $<_{e}$ has a topological property that makes it unique among other standard relation.

We will use the natural topology on the set of valuations coming from the identification of a valuation with the characteristic function of a set of propositional variables. In other words, each valuation $N$ is viewed as a function $N: \operatorname{Var} \rightarrow\{0,1\}$. The collection of all such functions is usually denoted by $\{0,1\}^{\operatorname{Var}}$. This set is endowed with the usual product topology where $\{0,1\}$ is given the discrete topology. We will assume that $V a r$ is countable, so $\{0,1\}^{V a r}$ is a metric space (in fact, homeomorphic to the classical Cantor space). The topology on $\{0,1\}^{V a r}$ is then defined by declaring $\bmod (\alpha)$ as the basic open sets for every formula $\alpha$ (in fact, $\bmod (\alpha)$ is also closed). We will regard $S$ as a topological space by using its subspace topology. The well known basic facts that will be needed about this topology are stated in the following lemma.

Lemma 4.1 (i) Let $N$ and $N_{i}$ with $i \geq 1$ be valuations. The following two conditions are equivalent: (a) $N_{i}$ converges to $N$. (b) for all formula $\alpha, N \models \alpha$ if and only if there is a $j$ such that $N_{i} \vDash \alpha$ for all $i \geq j$.

(ii) $A$ set $F \subseteq S$ is closed in $S$ iff given $N_{i} \in F$ converging to a normal valuation $N$, then $N \in F$.

(iii) If $F \subseteq S$ is closed in $S$ and $N \in S \backslash F$, then there is a formula $\alpha$ such that $N \models \alpha$ and $P \forall \neq \alpha$ for all $P \in F$.

(iv) Let $C$ be a set of formulas and $V \subseteq \bmod (C)$. Then $T h(V)=C n(C)$ iff $V$ is topologically dense in $\bmod (C)$ (i.e. for all $M \in \bmod (C)$ and all formula $\alpha$ with $M \models \alpha$, there is $N \in V$ such that $N \models \alpha$ ).

It is convenient to have a quick way of checking when an injective representation is in fact standard. The following lemma will be useful.

Lemma 4.2 Let $<$ be a relation over $S$ representing $\uparrow$.

(i) If $N \notin \min (\bmod (\alpha) \cap S,<)$ and $N \models C(\alpha)$, then there is a sequence $N_{i} \in \min (\bmod (\alpha) \cap S,<)$ converging to $N$.

(ii) < is standard iff $\min (\bmod (\alpha) \cap S,<)$ is topologically closed for all $\alpha$. In particular, if $\min (\bmod (\alpha) \cap S,<)$ is finite for all $\alpha$, then $<$ is standard.

Proof: From 4.1(ii) we have that $\bmod (C(\alpha))$ is closed and by $4.1($ iv $)$ we have that $\min (\bmod (\alpha) \cap$ $S,<)$ is dense in $\bmod (C(\alpha)$. From this the result follows.

We will present next an example of a consequence relation that has an injective model but does not satisfy WDR. In particular, by 3.4 this consequence relation does not have a standard representation. This result stands in contrast to what happens when the language is finite (see $3.12)$. 
Example 4.3 (A preferential relation not satisfying WDR and with an injective model) Let $\left\{p_{1}, p_{2}, \cdots, p_{n}, \cdots\right\}$ denote the set of propositional variables. Let $P$ be the valuation identically equal to one, i.e. $P \models p_{i}$ for all $i$. Let $Q$ be the valuation satisfying $Q \models p_{1}$ and $Q \models \neg p_{i}$ for $i>1$. Let $N$ be the valuation identically equal to zero, that is to say, $N \models \neg p_{i}$ for all $i$. Let $N_{i}$ and $M_{i}$ be such that $N_{i} \models \neg p_{1} \wedge \cdots \wedge \neg p_{i}$ and $N_{i} \models p_{j}$ for all $j>i ; M_{i} \vDash \neg p_{1} \wedge \cdots \wedge \neg p_{i} \wedge p_{i+1} \wedge \neg p_{i+2}$ and $M_{i} \models p_{j}$ for all $j>i+2$. Notice that both sequences converge to $N$.

We define a strict order $\prec$ over $S=\left\{N, P, Q, N_{i}, M_{i}\right\}$ by letting $P \prec N, Q \prec N, P \prec N_{i}$, $Q \prec M_{i}, N_{i} \prec N$ and $M_{i} \prec N$. Let $\sim$ be the preferential consequence relation defined by $(S, \prec)$. It is easy to check that $S$ is the collection of all normal valuations w.r.t. $\sim$. First we prove that every valuation in $S$ is normal. Note that $\min \left(\bmod \left(\neg p_{1}\right) \cap S, \prec\right)=\left\{N_{i}, M_{i}\right\}$ so $N_{i}$ and $M_{i}$ are normal and since $\bmod \left(C\left(\neg p_{1}\right)\right)$ is closed then $N \models C\left(\neg p_{1}\right)$. Notice that $N \notin \min \left(\bmod \left(\neg p_{1}\right) \cap S, \prec\right)$ and therefore $\prec$ is not standard. It is not difficult to see that $P \models C\left(p_{1} \wedge p_{2}\right)$ and $Q \models C\left(p_{1} \wedge \neg p_{2}\right)$. Conversely, suppose that $R \vDash C(\alpha)$. We want to show that $R \in S$. We know that $C(\alpha)=$ $T h(\min (\bmod (\alpha) \cap S))$. By 4.2 there exists a sequence $R_{i} \in \min (\bmod (\alpha) \cap S)$ converging to $R$. But it is easy to see that $S$ is closed, so $R \in S$.

We will show that $\sim$ does not satisfies WDR. For this end, it suffices to find two formulas $\alpha$ and $\beta$ such that $N \models C(\alpha) \cup C(\beta)$ but $N \not \models C(\alpha \vee \beta)$. Let $\alpha=\neg p_{1} \vee\left(p_{1} \wedge \neg p_{2}\right)$ and $\beta=\neg p_{1} \vee\left(p_{1} \wedge p_{2}\right)$. It is easy to verify that

$$
\begin{aligned}
\min (\bmod (\alpha) \cap S, \prec) & =\{Q\} \cup\left\{N_{i}: i \geq 1\right\} \\
\min (\bmod (\beta) \cap S, \prec) & =\{P\} \cup\left\{M_{i}: i \geq 1\right\} \\
\min (\bmod (\alpha \vee \beta) \cap S, \prec) & =\{P, Q\} .
\end{aligned}
$$

Therefore $N \models C(\alpha) \cup C(\beta)$, but $N \not \models C(\alpha \vee \beta)$.

We will introduce next a property that $<_{e}$ has and in fact it is the unique standard relation (with this property) that represents $\sim$.

Definition 4.4 Let $<$ be a binary relation over $S$, we will say $<$ is downward-closed is for all $N$ in $S$ the set $\{M \in S: M<N\}$ is (topologically) closed in $S$.

Lemma 4.5 Let $\sim$ be a consequence relation. Then $<_{e}$ is downward-closed.

Proof: Let $N, M, M_{i}$ be normal valuations with $M_{i}$ converging to $M$. Suppose that $M_{i}<_{e} N$ for all $i$. We will show that $M<_{e} N$. Let $\alpha$ be a formula such that $N \models C(\alpha)$, then by assumption $M_{i} \vDash \neg \alpha$. Since $M_{i}$ converges to $M$, then $M \models \neg \alpha$, i.e. $M<_{e} N$.

Lemma 4.6 Let $\sim$ be a consequence relation. Suppose that $<$ is a standard relation that represents $\sim$. If $<$ is downward-closed then $<=<_{e}$.

Proof: ¿From 3.5 we already know that $<\subseteq<_{e}$. For the other direction, let $N, M$ be normal valuations such that $M \nless N$. We will show that $M \nless_{e} N$. Since $F=\{P \in S: P<N\}$ is closed 
and $M \notin F$, then by 4.1 (iii) there is a formula $\alpha$ such that $M \models \alpha$ and $P \not \neq \alpha$ for all $P \in F$. Let $\beta$ be such that $N \vDash C(\beta)$. It suffices to show that $N \models C(\alpha \vee \beta)$. Since < is standard and represents $\sim$, then $N \in \min (\bmod (\beta) \cap S,<)$. Hence $P \not \notin \beta$ for all $P<N$. On the other hand, by the choice of $\alpha$, we also have that $P \not \forall \alpha$ for all $P<N$. Therefore $N \in \min (\bmod (\alpha \vee \beta) \cap S,<)$ and since < represents $\sim$ then $N \models C(\alpha \vee \beta)$.

From the previous results we immediately get the following

Theorem 4.7 Let $\sim$ be a preferential relation satisfying WDR. Then $<_{e}$ is the unique downwardclosed standard relation that represents $\uparrow$.

A valuation $N \in S$ is said to be isolated in $S$, if there is a formula $\alpha$ such that $\bmod (\alpha) \cap S=$ $\{N\}$. We will say that $S$ is discrete if every $N \in S$ is isolated in $S$. These notions correspond to the topological notion of an isolated point and discrete space. In particular, every finite set is discrete. In every discrete space the only converging sequences are the eventually constant sequences, therefore every relation over a discrete space is trivially downward-closed. On the other hand, by using the same argument as in the proof of 3.11 it can be easily checked that if $S$ is discrete and $\sim$ satisfies WDR, then $<_{e}$ is transitive. Moreover, by 4.2(i) we have also that any injective model defined on a discrete set is necessarily standard. Thus we have the following generalization of an analogous result known for finite languages.

Corollary 4.8 Let $\sim$ be a preferential consequence relation satisfying WDR. If the collection of normal valuations is discrete, then $<_{e}$ is the unique (and in fact standard) order representing $\sim$.

The following result might be known but it is now quite easy to show

Corollary 4.9 Let $\sim$ be a rational relation. Then $<_{e}$ is the unique standard modular order representing $\vdash$.

Proof: It suffices to show that every modular standard order representing $\sim$ is downward-closed. Let < be such modular relation and $M, N, N_{i}$ be normal valuations with $N_{i}$ converging to $N$ and $N_{i}<M$ for all $i$. Let $\alpha, \beta$ be formulas such that $M \models C(\alpha)$ and $N \models C(\beta)$. It suffices to show that $M \notin C(\alpha \vee \beta)$. Since in this case, there must exists a normal valuation $P<M$ such that $P \models \beta$. Since $N \models C(\beta)$ and $<$ is modular, standard and represents $\sim$ then $N<M$. To see that $M \not \models C(\alpha \vee \beta)$ we need to show that $M \notin \min (\bmod (\alpha \vee \beta) \cap S,<)$. Since $N \models \beta$ and $N_{i}$ converges to $N$, then there is (in fact, infinitely many) $i$ such that $N_{i} \vDash \beta$. Since $N_{i}<M$, then $M$ is not $\operatorname{minimal}$ in $\bmod (\alpha \vee \beta)$.

Since having a standard representation is a more restrictive condition we expected that it might imply that in this case $<_{e}$ should be transitive. In other words, if $\sim$ admits a standard representation (in particular, WDR holds) then $<_{e}$ must be transitive (and thus it would be a standard order representing $\sim$ ). Our second example-shows that this is not the case. This example will be used later also to show that in spite of the fact that $<_{e}$ might not be transitive it provides a very good representation of $\sim$ (even in some cases where other methods do not work). 
Example 4.10 A preferential relation $\sim$ with a standard model (in particular WDR holds) and $<_{e}$ not transitive

Let $\left\{p_{1}, p_{2}, \cdots, p_{n}, \cdots\right\}$ denote the set of propositional variables. We will define valuations $N, M$, $P, N_{i}$ and $M_{i}$ (for $i \geq 1$ ) viewing them as characteristic functions (i.e. as sequences of 0 and 1 ):

$$
\begin{aligned}
M_{i} & =\langle 0,0, \cdots, 0,1,1,1, \cdots, 1, \cdots\rangle & & \text { It starts with } i \text { ceros and then follows only } 1 \text { 's } \\
N_{i} & =\langle 0,0, \cdots, 0,1,0,1, \cdots, 1, \cdots\rangle & & \text { It starts with } i \text { ceros, then } 1,0 \text { and then follows only } 1 \text { 's } \\
P & =\langle 1,0, \cdots,> & & 1,0 \text { periodically repeated. } \\
M & =\langle 0,0, \cdots,\rangle & & \text { Only } 0 \text { 's } \\
N & =\langle 1,1, \cdots,\rangle & & \text { Only } 1 \text { 's }
\end{aligned}
$$

The order among this valuation is the transitive closure of the following pairs

$$
\begin{aligned}
N_{i} & <M_{i} \\
N_{i} & <N_{i+1} \\
M_{i} & <P \\
N & <M
\end{aligned}
$$

In particular we have that $N_{i}<P$ and also that $N_{i}<N_{j}$ and $N_{i}<M_{j}$ for all $i<j$. Notice that $M \nless P$. Let $S=\{N, M, P\} \cup\left\{N_{i}, M_{i}: i \geq 1\right\}$. Since < is clearly wellfounded then it is smooth. Let $\sim$ be the preferential relation defined by $(S,<)$. We claim that $S$ is the collection of normal valuation w.r.t. $\sim$. First, we show that the elements of $S$ are normal. Notice that every valuation isolated in $S$ is clearly normal. Since $M$ is the only not isolated point of $S$ it suffices to check that $M$ is a normal valuation. In fact, it is easy to verify that $M \in \min \left(\bmod \left(\neg p_{1}\right) \cap S,<\right)$. Conversely, suppose $R \models C(\alpha)$. We want to show that $R \in S$. To see that it is enough to prove that $\min (\bmod (\alpha) \cap S,<)$ is finite for every formula $\alpha$ and then we apply 4.2 . This also shows that $<$ is standard. Suppose that $\alpha$ uses only the letters $p_{1}, \cdots, p_{s}$. We consider two cases: (a) $\min (\bmod (\alpha) \cap S,<) \subset\{M, N, P\}$. In this case we are obviously done. (b) Suppose that for some $i$ either $N_{i} \models \alpha$ or $M_{i} \vDash \alpha$. If $N_{i} \models \alpha$ for some $i$, then it is easy to verify that

$$
\min (\bmod (\alpha) \cap S,<) \subset\{M, N\} \cup\left\{N_{j}, M_{j}: j \leq i\right\}
$$

and we will be done. Suppose then that $M_{i} \models \alpha$ for some $i$. Let $\gamma=\neg p_{1} \wedge \neg p_{2} \wedge \cdots \wedge \neg p_{s}$, then $N_{i}, M_{i} \models \gamma$ for all $i \geq s$. Observe that if $M_{i} \models \alpha$ for some $i \geq s$, then $\gamma \vdash \alpha$, thus $N_{s} \models \alpha$ and therefore by (4) we are done. From this it follows that $\min (\bmod (\alpha) \cap S,<)$ is finite for all $\alpha$.

Since $<$ is standard then from 3.4 we know that $\sim$ satisfies WDR and therefore by $3.10<_{e}$ is also a standard relation representing $\sim$. By 3.5 we have that $<\subseteq<_{e}$. However, $<_{e}$ is not transitive. We have that $N<_{e} M$ (as $N<M$ ) and we claim that $M<_{e} P$ but $N \Varangle_{e} P$. In fact, it is easy to check that $N, P \models C\left(p_{1}\right)$ and therefore $N \nless_{e} P$. On the other hand, $M_{i}$ converges to $M, M_{i}<P$ and since $<_{e}$ is downward-closed (by 4.5) then $M<_{e} P$.

To finish this section we will compare $<_{e}$ with the relation $<_{s}$ defined by Freund [2]. In particular, we will show that under some conditions (for instance if DR holds) $<_{e}$ is equal to $<_{S}$. Let $\sim$ be a preferential relation. We say that $\alpha$ is $\sim$-consistent if $\alpha \not \chi \perp$. The trace of a formula $\alpha$ is denote by $\alpha^{+}$and is defined as the set of all formulas $\beta$ such that $\alpha \vee \neg \beta \sim \beta$. The relation $<_{S}$ is defined over $S$ by

$$
M<_{S} N \Longleftrightarrow \forall \alpha \sim \text {-consistent }\left(N \models \alpha^{+} \Rightarrow M \not \models \alpha\right)
$$


For $\sim$ preferential, Freund showed that $<_{S}$ is transitive and irreflexive and also that $C(\alpha)=$ $C n\left(\{\alpha\} \cup \alpha^{+}\right)$for all $\alpha$. Now it is easy to verify that $<_{S} \subseteq<_{e}$ and that $<_{S}$ is a downward-closed relation.

A consequence relation is said to have the $\left({ }^{* *}\right)$ property if the following holds for every pair of $\sim$-consistent formulas $\alpha$ and $\beta$ :

$$
C(\alpha \vee \beta)=C n\left(\alpha^{+} \cup \beta^{+} \cup\{\alpha \vee \beta\}\right)
$$

The $\left(^{* *}\right)$ property seems to be tailor-made for getting part (i) of the following result

Theorem 4.11 (Freund [2]) (i) A preferential relation $\sim$ has the $\left(^{* *}\right.$ ) property iff $<_{S}$ is a standard order representing $\sim$.

(ii) Every disjunctive relation has the $\left(^{* *}\right)$ property.

(iii) The $\left(^{* *}\right)$ property implies WDR and they are equivalent when the language is finite.

(iv) DR is strictly stronger than WDR.

Since $<_{S}$ is downward-closed then from 4.6 and the previous theorem we conclude that $<_{e}=<_{S}$ for every preferential relation with the $\left({ }^{* *}\right)$ property (notice, that in this case $<_{e}$ is transitive). To complete the picture we have the following

Proposition 4.12 The $\left(^{* *}\right)$ property is strictly stronger than WDR. Moreover, there is a preferential relation represented by $<_{e}$ but not by $<_{S}$.

Proof: We will show that the consequence relation $\sim$ given in 4.10 does not have the $\left({ }^{* *}\right)$ property. Recall that $\sim$ was defined by a strict order that in fact is a standard model of $\sim$. In particular, $\sim$ satisfies WDR. Since $\sim$ is preferential then $<_{S}$ is transitive. But $<_{e}$ is not transitive, thus $<_{e} \neq<_{S}$. Therefore, $\sim$ does not have the $\left(^{* *}\right.$ ) property (otherwise $<_{S}$ would be equal to $<_{e}$ ). Moreover, by part (i) in Freund's theorem we conclude that $<_{S}$ does not represent $\sim$, but by $3.10<_{e}$ does (even though $\left(S,<_{e}\right)$ is not a standard model of $\sim$ because it is not transitive).

A final question: is there a postulate that characterize when a preferential relation has an injective model or a standard model? By the example 4.3 we know that WDR is not a necessary condition to have an injective model. The example 4.10 shows that the $\left({ }^{* *}\right)$ property is not a necessary condition (but it is sufficient) to have a standard model. None of our examples have ruled out that WDR suffices to obtain an standard model. Given a preferential relation $\sim$ satisfying WDR by 3.5 we know that any (if it exists) standard order representing $\sim$ has to be contained in $<_{e}$. Thus we have to remove from $<_{e}$ some pairs in order to make it transitive. We have tried (so far without success) the following strategy to get an injective (hopefully standard) model of $\sim$ : start with $<_{e}$ and remove all instances of non transitivity and get $<_{e}^{*} \subset<_{e}$. It is quite curious that this process indeed leads to a transitive relation. In principle, one would expect that when a pair is removed, then other instances of non transitivity might appear, but this is not the case with $<_{e}$. However, we have not been able to show that this "pruned" relation $<_{e}^{*}$ still represents $\sim$ (we even don't know if $<_{e}^{*}$ is still smooth). These two families of consequence relations seems so complex that we will not be surprised if there is no such a characterization (at least in terms of the type of postulates used so far to classify consequence relations). 
Acknowledgments: Partial support for Carlos Uzcátegui was provided by a CDCHT-ULA (Venezuela) grant. This work was initiated while he was visiting the Laboratoire d'Informatique Fondamentale de Lille (LIFL), France. He would like to thank LIFL for the financial assistance and facilities they provided.

The final version of this paper was done when Ramón Pino Pérez was visiting the Mathematics department of University of Los Andes (Venezuela). He would like to thank the UNESCO's TALVEN program and the Mathematics department of ULA for the partial support they provided.

\section{References}

[1] H. Bezzazi and R. Pino Pérez. Rational transitivity and its models. In IEEE Computer Society Press, editor, Proceedings of the twenty-sixth International Symposium on Multiple-Valued Logic., pages 160-165, Santiago de Compostela, Spain, May 29-31, 1996.

[2] M. Freund. Injective models and disjunctive relations. J.Logic Computat, 3:231-247, 1993.

[3] M. Freund, D. Lehmann, and P. Morris. Rationality, transitivity and contraposition. Artificial Intelligence, 52:191-203, 1991.

[4] P. Gärdenfors and D. Makinson. Nonmonotonic inferences based on expectations. Artificial Intelligence, 65:197-245, 1994.

[5] R. Pino Pérez H. Bezzazi, D. Makinson. Beyond rational monotony: some strong non-horn rules for nonmonotonic inference relations. J. Logic and Computation, To appear.

[6] S. Kraus, D. Lehmann, and M. Magidor. Nonmonotonic reasoning, preferential models and cumulative logics. Artificial Intelligence, 44(1):167-207, 1990.

[7] D. Lehmann and M. Magidor. What does a conditional knowledge base entail? Artificial Intelligence, 55:1-60, 1992.

[8] J. Lobo and C. Uzcátegui. Abductive consequence relations. Artificial Intelligence, 89(12):149-171, 1997.

[9] D. Makinson. General patterns in nonmonotonic reasoning. In C. Hogger D. Gabbay and J. Robinson, editors, Handbook of Logic in Artificial Intelligence and Logic Programming, volume III, Nonmonotonic Reasoning and Uncertain Reasoning. Oxford University Press, 1994.

[10] R. Pino Pérez and C. Uzcátegui. Jumping to explanation vs jumping to conclusions. Pre-print.

Lab. d'Informatique Fondamentale de Lille. U.A. 369 du CNRS. Université de Lille I. 59655 Villeneuve d'Ascq, France.

e-mail: pino@lifl.fr

Departamento de Matemáticas. Facultad de Ciencias. Universidad de Los Andes. Mérida 5101. Venezuela

e-mail address: uzca@ciens.ula.ve 